\title{
Model-oriented Availability Analysis of Optical GEO-Ground Links
}

\author{
Nicolas Perlot ${ }^{\text {a }}$, Josep Perdigues-Armengol ${ }^{\text {b }}$ \\ ${ }^{a}$ Institute of Communication and Navigation, DLR, 82234 Wessling, Germany \\ ${ }^{\mathrm{b}}$ ESA-ESTEC, Keplerlaan 1, 2200 AG Noordwijk, Netherlands
}

\begin{abstract}
The impact of clouds on optical Earth-space links is assessed by means of theoretical analyses. The spacecraft is assumed geostationary so that a stationary model can be considered for the link availability. Binary and continuous attenuations are analysed separately. With a network of ground stations, both spatial diversity and multiplexing are considered. The impact of the ground-station network size and spatial correlation on the availability and throughput is studied. Using a continuous attenuation model, it is shown that an adaptive data rate essentially mitigates thin clouds (on the order of $10-\mathrm{dB}$ attenuation).
\end{abstract}

Keywords: Free-space optical communications, Satellite communications, MIMO systems, Channel diversity.

\section{INTRODUCTION}

Because optical frequencies are highly attenuated by clouds, optical communications through the atmosphere must mitigate clouds to become a reliable technology. The fact that cloud attenuation can easily be higher than $100 \mathrm{~dB}$ makes pertinent the use of a binary attenuation model with the two states "covered" and "clear" [1][2][3]. However, some thin clouds can have an attenuation less than $30 \mathrm{~dB}$. For these weaker attenuations, a performance evaluation should rely on a continuous-attenuation model.

Considering a geostationary satellite throughout the paper, attenuation is assumed to be a stationary random variable. This stationarity is an approximation if one considers e.g. seasonal variations, but the approximation can be alleviated e.g. if it is applied to one season. Additionally, the paper focuses on point-to-point Earth-space links. For such scenarios, the deployment of a network of ground stations (GS) provides spatial diversity with respect to cloud coverage [4]. Based on a GS network, two transmission concepts are considered: GS selection combining (GS-SC) and GS multiplexing (GSMUX). These are depicted in Figure 1.
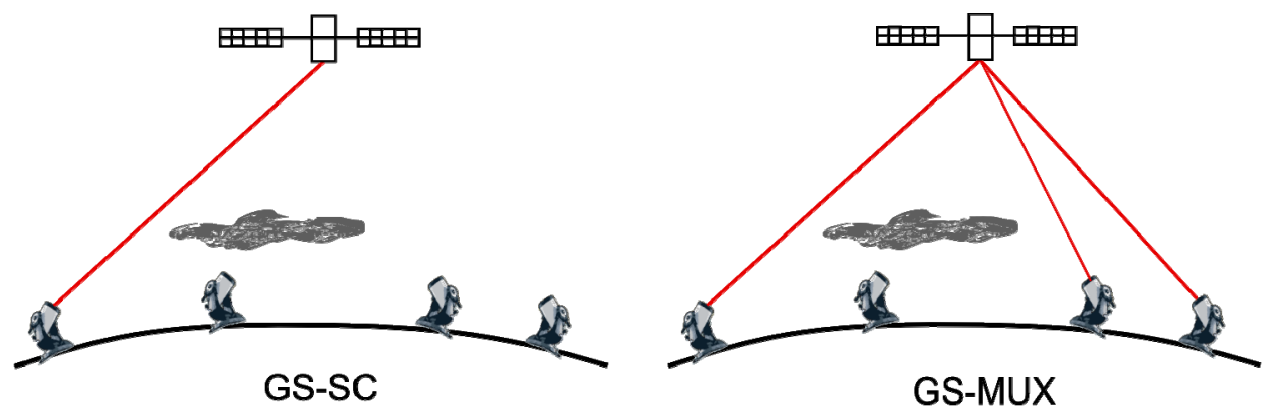

Figure 1: Two considered transmission techniques based on a network of ground stations (GSs): selection combining (GSSC) and multiplexing (GS-MUX).

Whereas GS-SC can significantly increase the link availability by switching the link to one of the available GSs, GSMUX can significantly increase the link throughput by establishing simultaneous links with all the available GSs. Several simultaneous links imply that the space segment bears several transmitters or receivers. On the space segment, we can think of three ways to accommodate several transceivers:

Free-Space Laser Communication Technologies XXIV, edited by Hamid Hemmati, Don M. Boroson, Proc. of SPIE Vol. 8246, 82460P - (C) 2012 SPIE · CCC code: 0277-786X/12/\$18 - doi: 10.1117/12.908706 
(1) one telescope with several transceivers distributed in the focal plane

(2) several telescopes on a spacecraft, with one transceiver per telescope

(3) several spacecraft with one or more terminals.

Between 2 GSs separated by $1000 \mathrm{~km}$, a GEO satellite typically sees an angle of $25 \mathrm{mrad}\left(1.5^{\circ}\right)$. The implementation of an array of transceivers in the focal plane, i.e. accommodation of type (1), is then conceivable although technically challenging.

For weak cloud attenuations, other types of mitigation may be employed. We will consider adaptive data rate (ADR) but will ignore adaptive transmit power. Adapting the transmit power according to the attenuation is attractive only when the link is not power limited (i.e. when transmitting the full power is not necessary), which is not the case in most optical space links.

The paper is organized as follows. In Section 2, we consider a binary attenuation model. In that context, we study the impact of the GS network size and spatial correlation on the link availability and throughput. The link availability is also assessed for the case of an airborne terminal. In Section 3, we consider a continuous attenuation model. Defining first an attenuation distribution for a single GS, we derive the combined transmitted power distribution of a GS network. The mean throughput is then derived for fixed and adaptive data rates. We briefly discuss the benefits of an ADR and then conclude.

\section{BINARY ATTENUATION MODEL}

\subsection{Link availability}

\section{Independent GSs with equal probabilities}

If the distance between any two GSs is much larger than the cloud correlation distance, the GSs can be assumed statistically independent. If additionally all GSs have the same cloud probability $p_{\text {cloud }}$, then the probability $P_{k, n}$ that $k$ stations among a total of $n$ stations are available at a given time is governed by the binomial distribution:

$$
P_{k, n}=\left(\begin{array}{l}
n \\
k
\end{array}\right) p_{\text {cloud }}^{n-k}\left(1-p_{\text {cloud }}\right)^{k}
$$

The link outage probability (LOP) is the probability that 0 station is available $(k=0)$ :

$$
L O P=p_{\text {cloud }}^{n} \text {. }
$$

So the number $n$ of stations can be expressed as a ratio of dB terms: $n=(L O P)_{d B} /\left(p_{c l o u d}\right)_{d B}$. For different $p_{\text {cloud }}$ values, Table 1 gives the number $n_{1 \_n i n e}$ of stations leading to an availability of $90 \%$ (i.e., a "1-nine" availability). Knowing the number $n_{1_{-} \text {nine }}$ for a 1-nine availability, we easily derive the number $n_{j_{-} \text {nine }}$ for a $j$-nine availability according to $n_{j_{-} \text {nines }}=j \times n_{1_{-} \text {nine }}$. In Figure 2, the number of GSs is shown for different availabilities.

\begin{tabular}{|c|c|c|c|c|}
\hline \multirow{2}{*}{$\begin{array}{c}\text { LOP } \\
(90 \% \text { availability })\end{array}$} & \multicolumn{4}{|c|}{$1 \mathrm{e}-1$} \\
\cline { 2 - 5 } & 0.32 & 0.47 & 0.63 & 0.75 \\
\cline { 2 - 5 }$p_{\text {cloud }}$ & $-5 \mathrm{~dB}$ & $-3.3 \mathrm{~dB}$ & $-2.0 \mathrm{~dB}$ & $-1.25 \mathrm{~dB}$ \\
\hline$n_{1 \text { _nine }}$ & 2 & 3 & 5 & 8 \\
\hline
\end{tabular}

Table 1: Number $n_{1 \_n i n e}$ of stations to reach an availability of $90 \%$ (a "1-nine" availability). Different $p_{\text {cloud }}$ values are considered. Independent stations with equal cloud probabilities are assumed. 


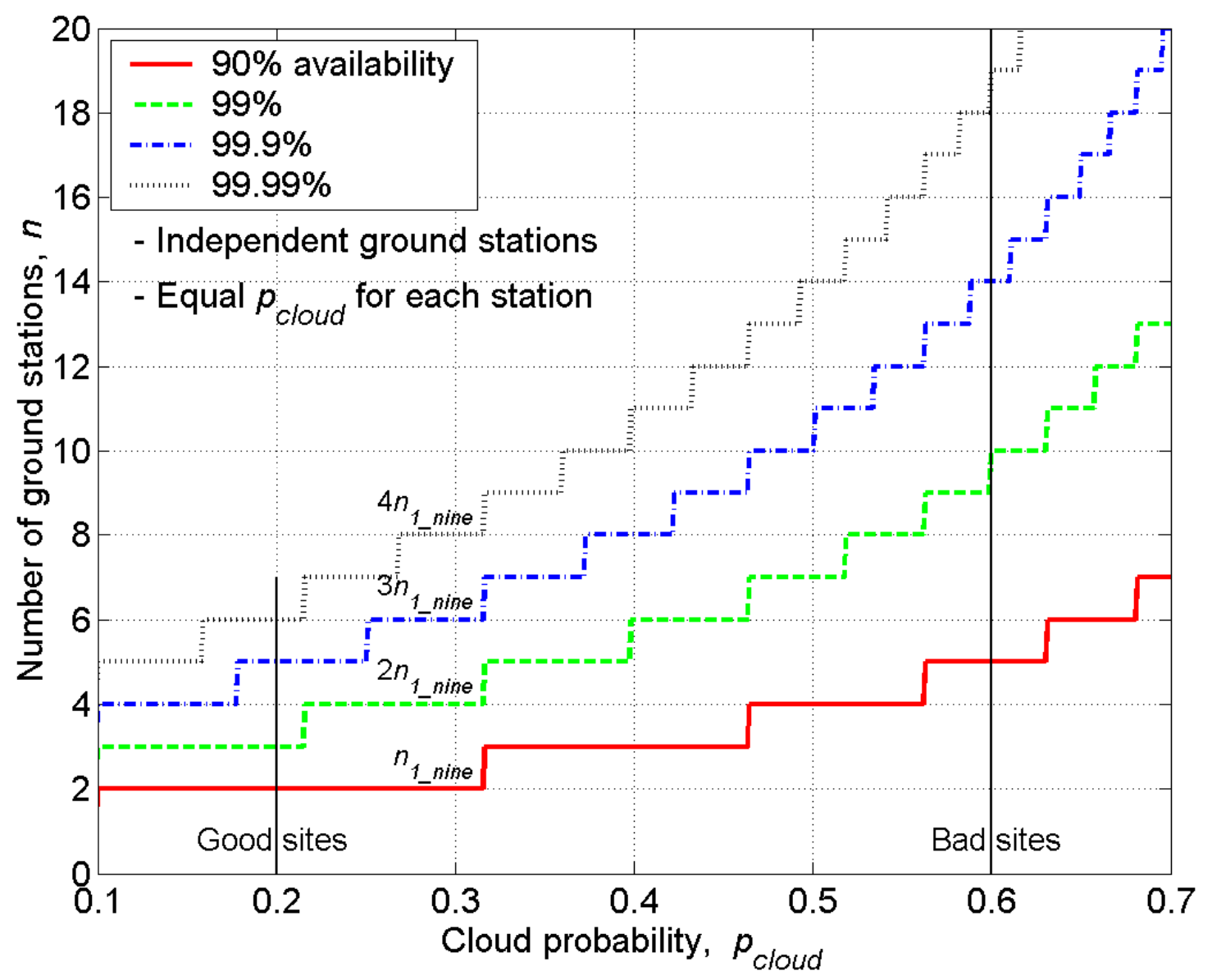

Figure 2: Number of GSs required to reach a given availability as a function of cloud probability. GSs are assumed independent and with equal cloud probability.

\section{Correlated GSs}

If the GSs are correlated, the analytical problem is too complex and one must resort to simulations of random processes. To generate correlated binary (Cloud/No-cloud) variables, we first generate the correlated Gaussian variables $Y_{1}, Y_{2}, \ldots$, $Y_{n}$. As shown in Figure 3 and Figure 4, we then operate a threshold transform to obtain correlated binary variables $Z_{1}, Z_{2}$, $\ldots, Z_{n}$. However, the threshold operation modifies the correlation between the variables. It is therefore necessary to premodify the correlation matrix of the Gaussian variables so that the obtained binary variables have the correct correlation matrix.

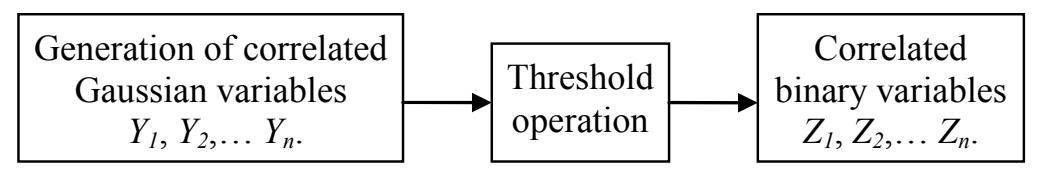

Figure 3: Procedure for generating correlated binary variables. 


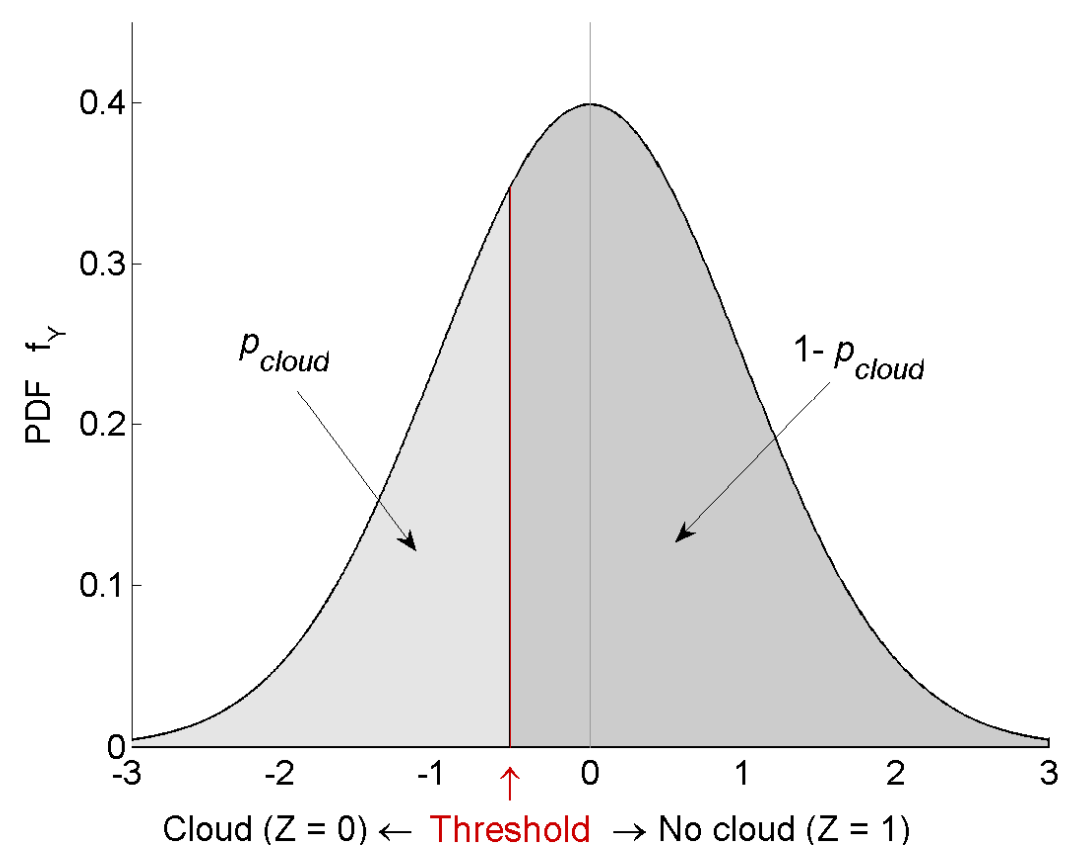

Figure 4: Threshold operation to transform Gaussian variable in binary variables

Measurements have shown that the cloud correlation coefficient $r_{Z}$ between two stations can be reasonably modelled by an exponential fall-off [5]:

$$
r_{Z}(d)=e^{-d / d_{0}}
$$

with $\quad d$ the distance between two GSs

$d_{0}$ the cloud correlation distance (typically, $300 \mathrm{~km}$ )

The correspondence between $r_{Y}$ and $r_{Z}$, the $Y$ and the $Z$ correlation coefficients, can be found by expressing the coverage correlation $\left\langle Z_{1} Z_{2}\right\rangle$ of two stations according to [6]

$$
\begin{aligned}
\left\langle Z_{1} Z_{2}\right\rangle & =\int_{-\infty}^{\infty} \int_{-\infty}^{\infty} Z_{1}\left(y_{1}\right) Z_{2}\left(y_{2}\right) f_{Y_{1}, Y_{2}}\left(y_{1}, y_{2}, r_{y_{1} y_{2}}\right) d y_{1} d y_{2} \\
& =\int_{\beta_{1}}^{\infty} \int_{\beta_{2}}^{\infty} f_{Y_{1}, Y_{2}}\left(y_{1}, y_{2}, r_{y_{1} y_{2}}\right) d y_{1} d y_{2}
\end{aligned}
$$

where $f_{Y_{1}, Y_{2}}\left(y_{1}, y_{2}, r_{y_{1} y_{2}}\right)$ is the joint Gaussian PDF of $Y_{1}$ and $Y_{2}$ at the two stations, and $\beta_{i}=\Phi^{-1}\left(p_{\text {cloud }, i}\right)$ with $\Phi^{-1}$ the standard Gaussian inverse CDF. For illustration purposes, we consider networks that form a grid on the Earth surface. Figure 5 shows a grid network of 9 ground stations with a minimum distance of $d_{x}=200 \mathrm{~km}$ between two stations. Figure 6 shows the LOP (i.e., probability that all stations are covered) of a grid network as a function of the number of stations. The cloud cover probability $p_{\text {cloud }}$ is the same for every station in the network. In Figure 7 , the probability distribution of the number of available stations among a grid network of $n=9$ stations. 


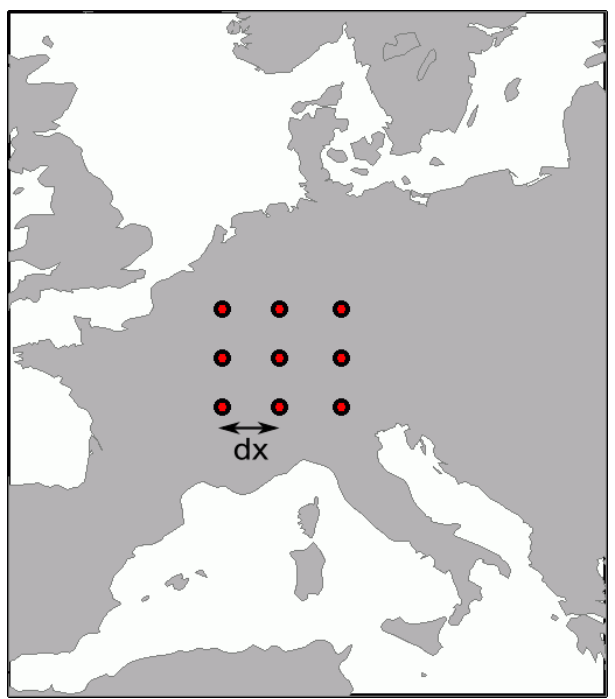

Figure 5: Grid network of 9 stations in central Europe with station interval $d_{x}=200 \mathrm{~km}$.

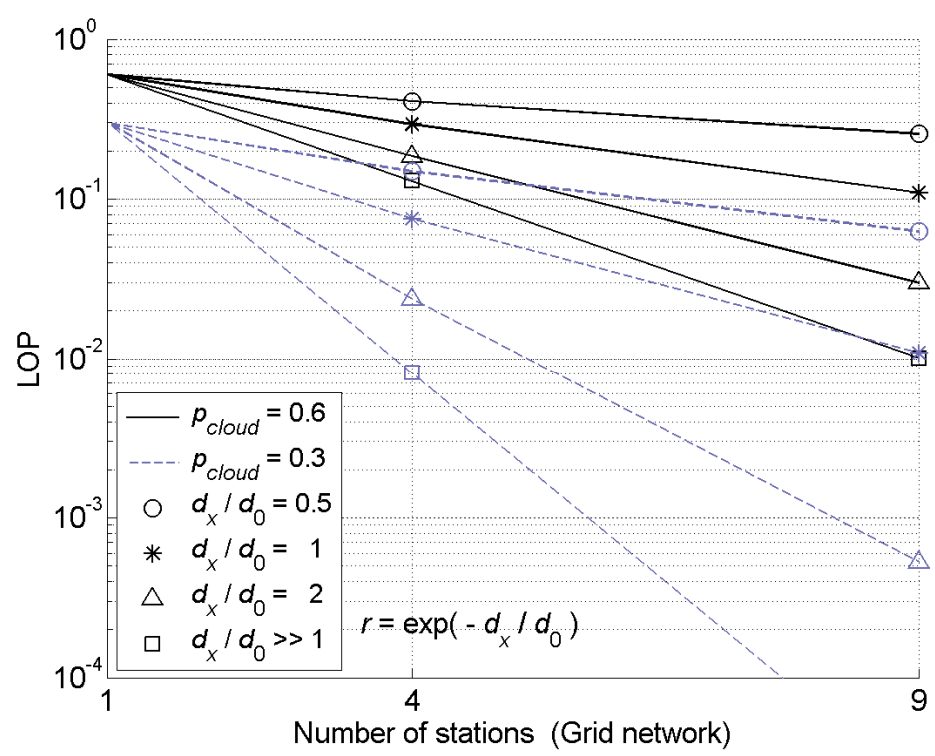

Figure 6: Link outage probability (LOP) of a grid network as a function of the number $n$ of stations. Two different $p_{\text {cloud }}$ values and four different spatial correlation values $r$ are considered.

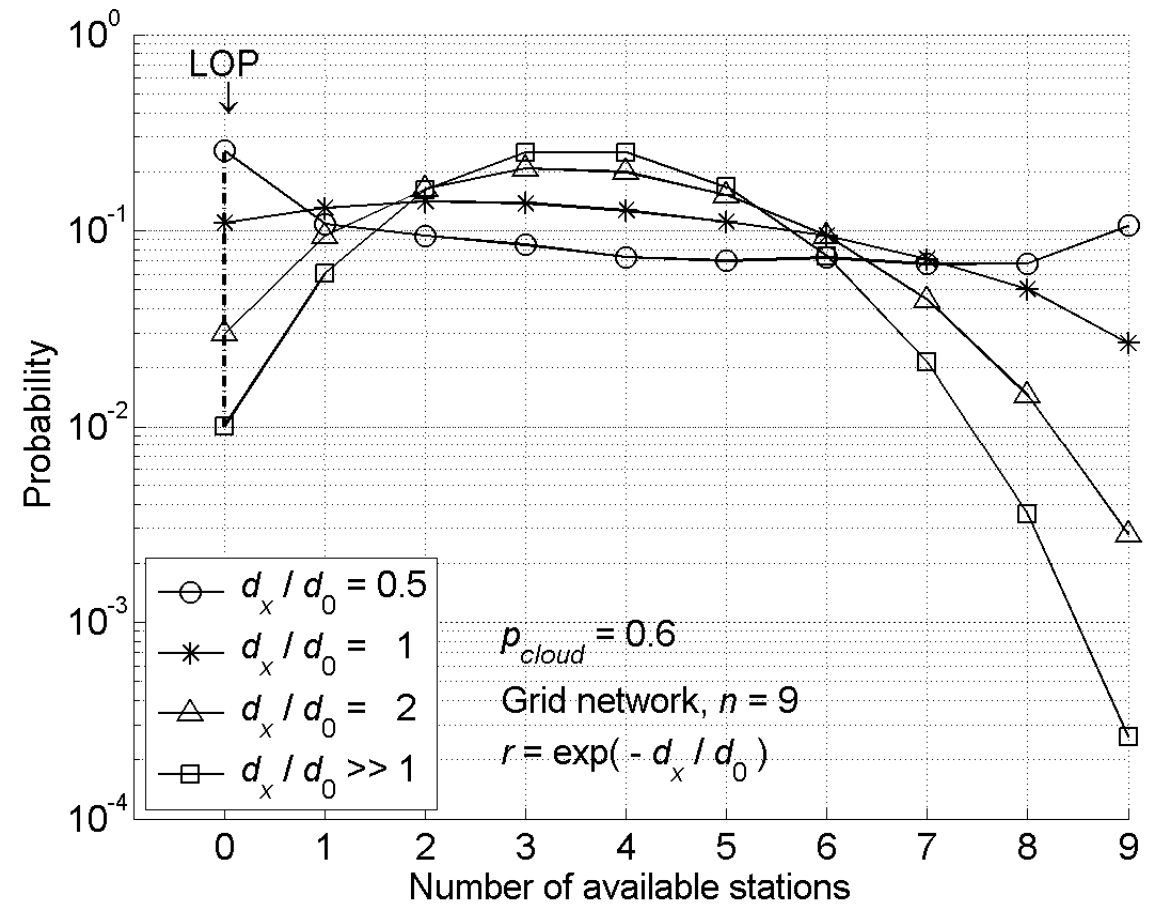

Figure 7: Probability distribution of the number of available stations among a grid network of $n=9$ stations. Probabilities of zero available station are indicated as link outage probabilities (LOP). four different spatial correlation values $r$ are considered. 


\subsection{Simultaneous availability of at least 2 GSs}

Once a GS network has been deployed, one may think about using this network to operate several links with several GSs simultaneously. Accordingly, several terminals (or transceivers) should be present onboard the satellite(s). The probability $L O P_{2 \_ \text {links }}$ that less than 2 stations are available is given by

$$
L O P_{2_{-} \text {links }}=\operatorname{Prob}(0 \text { station available })+\operatorname{Prob}(1 \text { station available }) .
$$

Assuming a binomial distribution for the number of available stations (see Eq. (1)), we have

$$
\begin{aligned}
L O P_{2 \_ \text {links }} & =p_{\text {cloud }}^{n}+n\left(1-p_{\text {cloud }}\right) p_{\text {cloud }}^{n-1} \\
& =p_{\text {cloud }}^{n}\left[1+n \frac{1-p_{\text {cloud }}}{p_{\text {cloud }}}\right] \\
& =L O P_{1 \_ \text {link }}\left[1+n\left(\frac{1}{p_{\text {cloud }}}-1\right)\right]
\end{aligned}
$$

Now, considering a high-availability network with e.g. $n>5$ and $p_{\text {cloud }}<0.5$, we have

$$
L O P_{2 \_ \text {links }}>L O P_{1 \_ \text {link }} \text {. }
$$

This means that a GS network dimensioned to provide a high availability to 1 link (with 1 GS) cannot provide a similar availability to 2 simultaneous links (with 2 GSs). For 2 links, the network would have to be extended.

\subsection{Mean throughput}

The main goal of implementing GS-MUX rather than a simple GS-SC is to increase the total data throughput of both upand downlinks. The mean number of available links (or GSs) in a GS-MUX configuration is independent of the level of correlation between the GSs and is equal to $n\left(1-p_{\text {cloud }}\right)$. Recalling that the availability of a GS-SC link is $1-p_{\text {cloud }}^{n}$ for the case of statistically independent GSs, and $1-p_{\text {cloud }}$ for the case of fully correlated GSs, the ratio of the GSMUX throughput, noted $T_{M U X}$, to the GS-SC throughput, noted $T_{S C}$, is thus

$$
\frac{T_{M U X}}{T_{S C}}= \begin{cases}\frac{n\left(1-p_{\text {cloud }}\right)}{1-p_{\text {cloud }}^{n}} & \text { if independence } \\ n & \text { if full correlation }\end{cases}
$$

This ratio is displayed in Figure 8. One observes that the throughput improvement of GS-MUX with respect to GS-SC increases with (i) the number $n$ of stations, (ii) the clear-sky probability $1-p_{\text {cloud }}$ of each GS and (iii) the level of correlation between the GSs. 


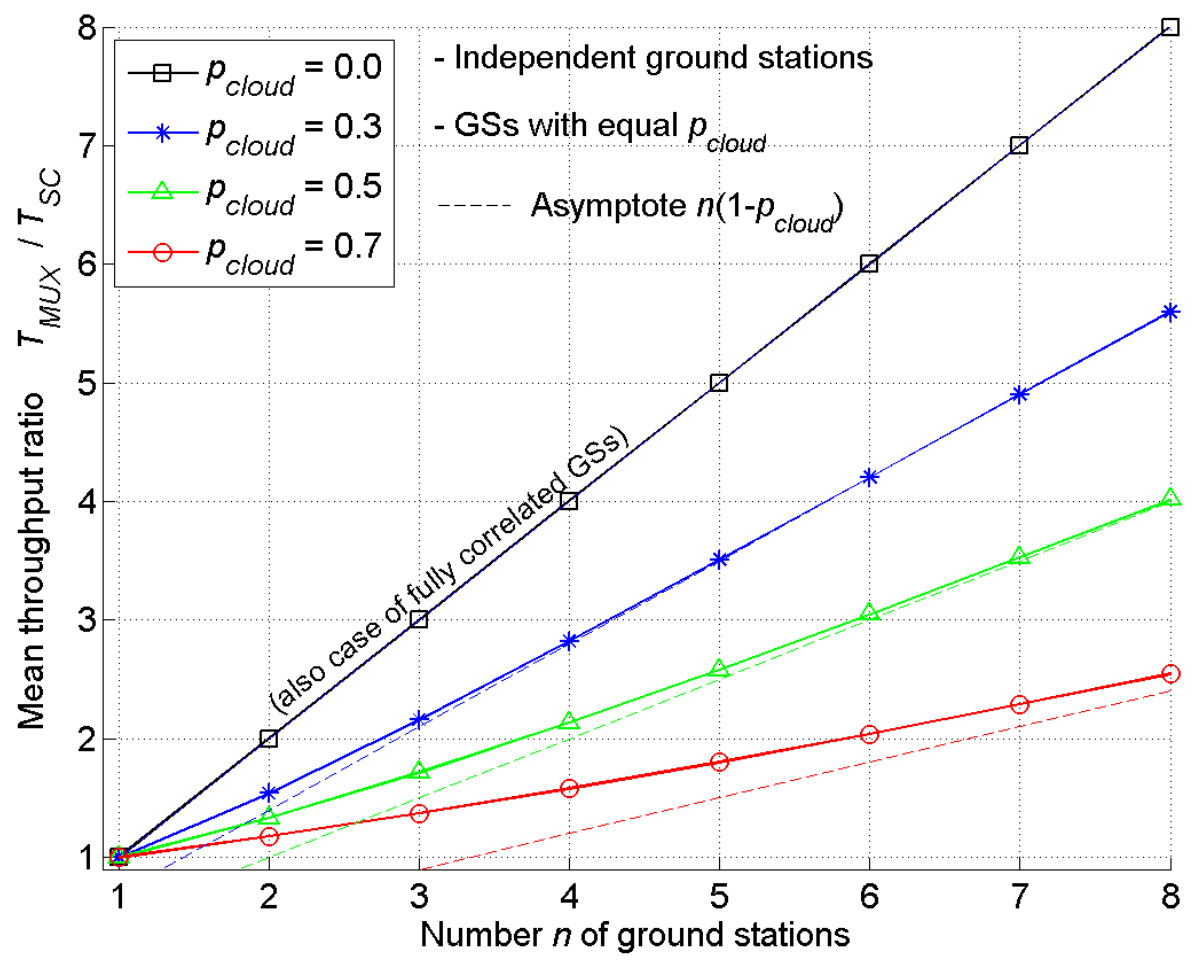

Figure 8: Throughput increase factor of GS-MUX with respect to GS-SC. Different numbers $n$ of GSs and different cloud probability $p_{\text {cloud }}$ are considered.

\subsection{Airborne Terminal}

Accommodating the Earth terminal on an aircraft rather than on ground can significantly improve the link availability [7]. Clouds can be as high as $13-\mathrm{km}$ altitude. However, most clouds are located below $6-\mathrm{km}$ altitude. Some statistics on low-, middle- and high-level clouds are available [8] and we propose a way to estimate the link availability as a function of terminal height. We consider the probability $p_{\text {cloud }, k}$ of cloud within an altitude level $k$. Table 2 gives examples of such probabilities for the region of Paris. We note $h_{k}, h_{k+1}$ the altitude limits of the level $k$.

\begin{tabular}{|l|l|l|l|l|}
\hline Altitude level & Low & Middle & High & \\
\hline$k$ & 1 & 2 & 3 & 4 \\
\hline$h_{k} \ldots h_{k+1}$ & $0 \ldots 2 \mathrm{~km}$ & $2 \ldots 6 \mathrm{~km}$ & $6 \ldots 13 \mathrm{~km}$ & $13 \ldots \infty \mathrm{km}$ \\
\hline$p_{\text {cloud }, k}$ & 0.40 & 0.25 & 0.15 & 0 \\
\hline
\end{tabular}

Table 2: Level cloud probabilities in the region of Paris (based on data of [8]).

We then assume that occurrences of clouds in different levels are statistically independent. The link availability $L A\left(h_{k}\right)$ for a terminal located at an altitude $h_{k}$ is thus

$$
L A\left(h_{k}\right)=\prod_{m=k}^{M}\left(1-p_{\text {cloud }, m}\right) .
$$

where $M=3$ according to our level decomposition. Based on the values of Table 2, the availabilities $L A\left(h_{k}\right)$ are shown in Figure 9. We observe that the link availability for a terminal at an altitude of $h=6 \mathrm{~km}$ is only $85 \%$. This can be improved by mitigating cirrus attenuation (e.g. with an adaptive data rate). 


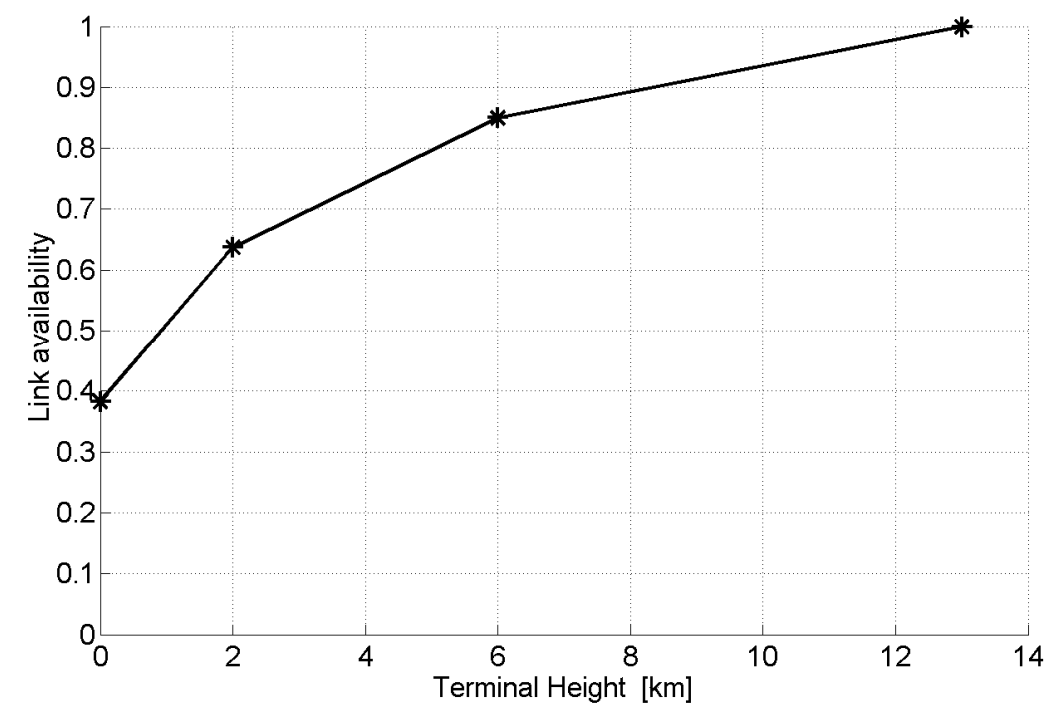

Figure 9: Link availability when the Earth terminal is airborne. Calculations are based on Eq. (9) and typical central-Europe statistics [8].

\section{CONTINUOUS ATTENUATION MODEL}

\subsection{Attenuation distribution (single GS)}

We consider an attenuation model at a GS based on the following random events:

$E_{1}$ : No cloud

$E_{2}$ : Only thin clouds

$E_{3}$ : One thick cloud is present (possibly superposed to other clouds)

These events are mutually exclusive and collectively exhaustive. We thus have

$$
\sum_{i=1}^{3} \operatorname{Prob}\left(E_{i}\right)=1
$$

and the PDF $f_{a}(a)$ of the attenuation $a$ is given by

$$
f_{a}(a)=\sum_{i=1}^{3} \operatorname{Prob}\left(E_{i}\right) \times f_{a}\left(a \mid E_{i}\right) .
$$

For illustration purposes, we consider an instance of $f_{a}(a)$ with values of $\operatorname{Prob}\left(E_{i}\right)$ as given by Table 3. The conditional PDFs $f_{a}\left(a \mid E_{i}\right)$ are modelled so that absolute attenuation values in $\mathrm{dB}$ within the event $E_{i}$ follow a lognormal distribution. The considered mean and standard deviation of each of these lognormal variables are also given in Table 3. Although values of Table 3 are estimated from properties of the clear sky and of clouds [KAR88] [CHU89], the attenuation PDF model is expected to strongly vary with the geographical location. For example, clear-sky attenuation will vary with the concentration of aerosols which in turn can be influenced by a volcanic activity. The total $f_{a}(a)$, as given in Eq. (11), is illustrated in Figure 10. The two elevations $90^{\circ}$ and $10^{\circ}$ are considered with the attenuation in $\mathrm{dB}$ at $10^{\circ}$ being about 5 times higher than that at $90^{\circ}$. 


\begin{tabular}{|l|c|c|c|}
\cline { 2 - 4 } \multicolumn{1}{c|}{} & Prob $\left(E_{i}\right)$ & $\begin{array}{l}\text { Att. mean (absolute } \\
\text { value) }\end{array}$ & $\begin{array}{l}\text { Att. } \\
\text { deviation }\end{array}$ \\
\hline $\boldsymbol{E}_{1}$ : No cloud & 0.5 & $0.8 \mathrm{~dB}$ & $0.18 \mathrm{~dB}$ \\
\hline $\boldsymbol{E}_{2}$ : Only thin clouds & 0.1 & $8 \mathrm{~dB}$ & $5.7 \mathrm{~dB}$ \\
\hline $\boldsymbol{E}_{3}$ : Thick cloud present & 0.4 & $300 \mathrm{~dB}$ & $340 \mathrm{~dB}$ \\
\hline
\end{tabular}

Table 3: Considered parameters for continuous attenuation model at $90^{\circ}$ elevation.

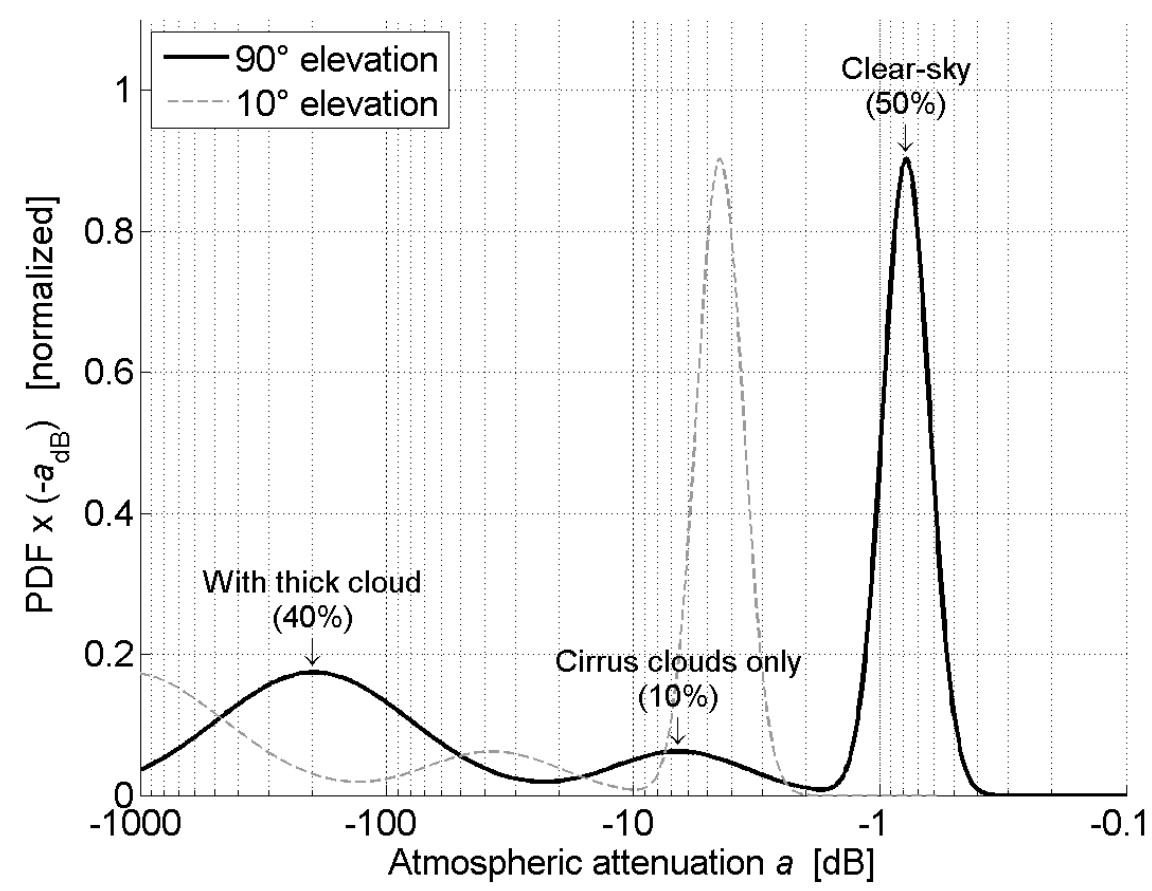

Figure 10: Probability distribution model for a continuous attenuation at a GS.

\subsection{Transmission distribution (multiple GSs)}

With $a_{j}$ the attenuation at the $j^{\text {th }} \mathrm{GS}$, the link attenuation in the case of GS-SC is

$$
a_{S C}=\max \left(a_{j}\right),
$$

whereas the cumulated attenuation associated with the cumulated link power of GS-MUX is

$$
a_{M U X}=\sum_{j} a_{j} .
$$

Invoking statistical independence between the GSs, the cumulative distribution function (CDF) of the signal attenuation in case of GS-SC is given by

$$
\begin{aligned}
F_{S C}(a) & =\prod_{j=1}^{n} F_{a_{j}}(a) . \\
& =F_{a}^{n}(a) .
\end{aligned}
$$


where in the second line we used our assumption of equal statistics for all GSs, i.e. $F_{a_{j}}(a)=F_{a}(a)$. The PDF of the cumulated signal attenuation in case of GS-MUX is given by

$$
f_{M U X}(a)=f_{a_{1}}(a) \otimes f_{a_{2}}(a) \otimes \cdots \otimes f_{a_{n}}(a) .
$$

where $\otimes$ denotes convolution. Considering the single-GS attenuation PDF $f_{a}(a)$ plotted in Figure 10, we use Eqs. (14) and (15) to plot in Figure 11 the CDF of the normalized transmitted power (equivalently, of the accumulated attenuation). If we now consider a given transmitted power as a level under which there is a link outage, the CDF curves of Figure 11 correspond to LOP curves.

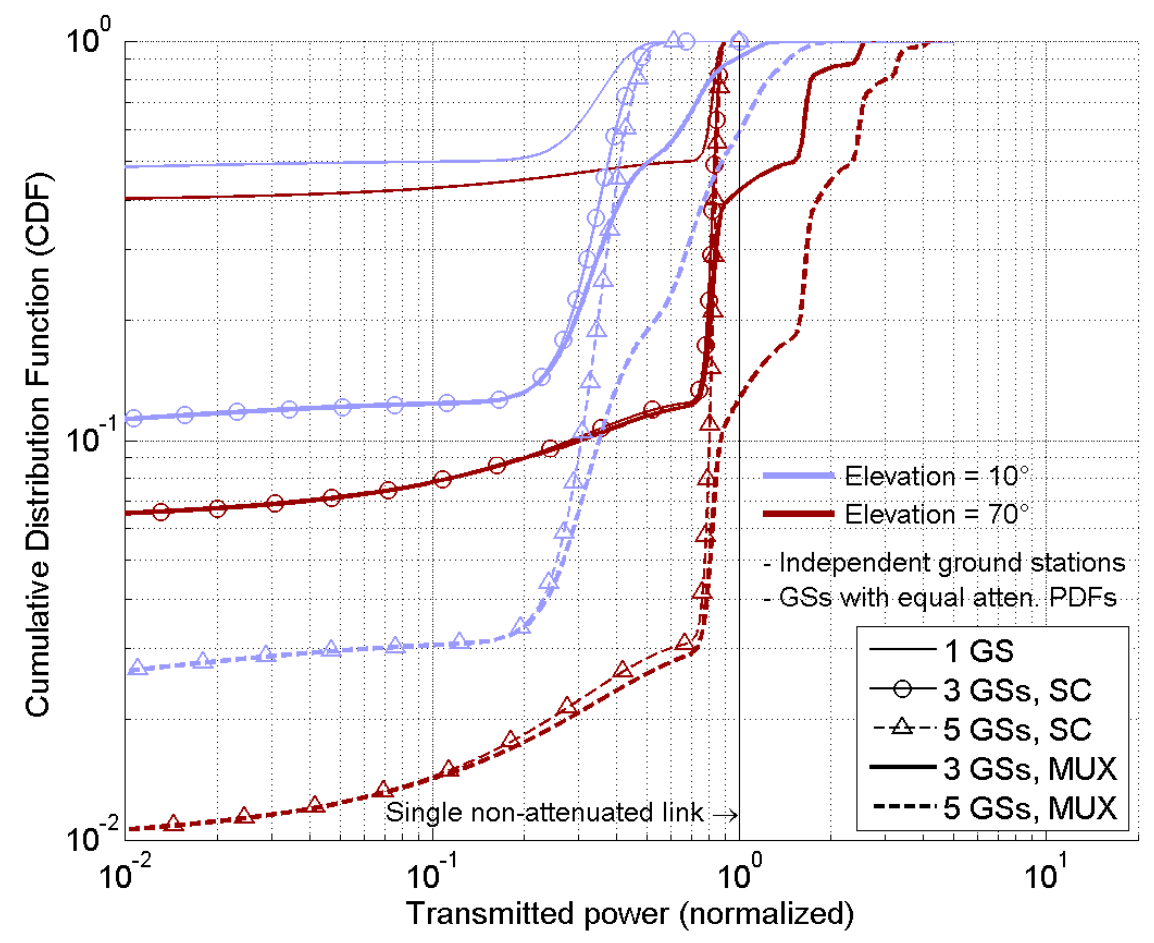

Figure 11: CDF of the transmitted power normalized to that of a single non-attenuated link. Equal attenuation PDFs and statistical independence are assumed for the GSs of a network.

\subsection{Mean throughput}

\section{Fixed data rate}

In the case of a fixed data rate, we consider the data rate $B_{\text {data,opt }}$ that maximizes the (mean) throughput. In general, the throughput $T\left(B_{\text {data }}\right)$ is given by the data rate $B_{\text {data }}$ multiplied by its associated availability $L A\left(B_{\text {data }}\right)$. The throughput $T_{F D R}$ for a fixed data rate is then

$$
\begin{aligned}
T_{F D R} & =\max \left[T\left(B_{\text {data }}\right)\right]_{B_{\text {data }}} \\
& =\max \left[B_{\text {data }} L A\left(B_{\text {data }}\right)\right]_{B_{\text {data }}}
\end{aligned}
$$

For the case of GS-SC, we can write

$$
T_{S C, F D R}=B_{\text {data,SC,opt }} \times L A_{S C, \text { opt }} .
$$


where both the optimum link availability $L A_{S C \text {,opt }}$ and the optimum fixed data rate $B_{\text {data,SC,opt }}$ depend on the number $n$ of GSs. The two different values of $B_{\text {data,SC,opt }}$ for 1 GS and for a 5-GS selection combining (SC) network are shown in Figure 12 where we assume:

- the proportionality of the data rate with the received power

- equal data-rate capabilities for each GS

- the same attenuation model $f_{a}(a)$ at $70^{\circ}$ for each GS

- statistical independence between the GSs

We observe in this example that the variation of the fixed data rate $B_{\text {data,SC, opt }}$ is small (about $5 \%$ ). When GS-MUX is implemented, the throughput derived from Eq. (16) is given by

$$
\begin{aligned}
T_{M U X, F D R} & =\sum_{j=1}^{n} B_{\text {data }, o p t, j} L A_{o p t, j} \\
& =n B_{\text {data }, \text { opt }} L A_{\text {opt }}
\end{aligned}
$$

where in the second line we have assumed equal attenuation statistics for all $n$ GSs.

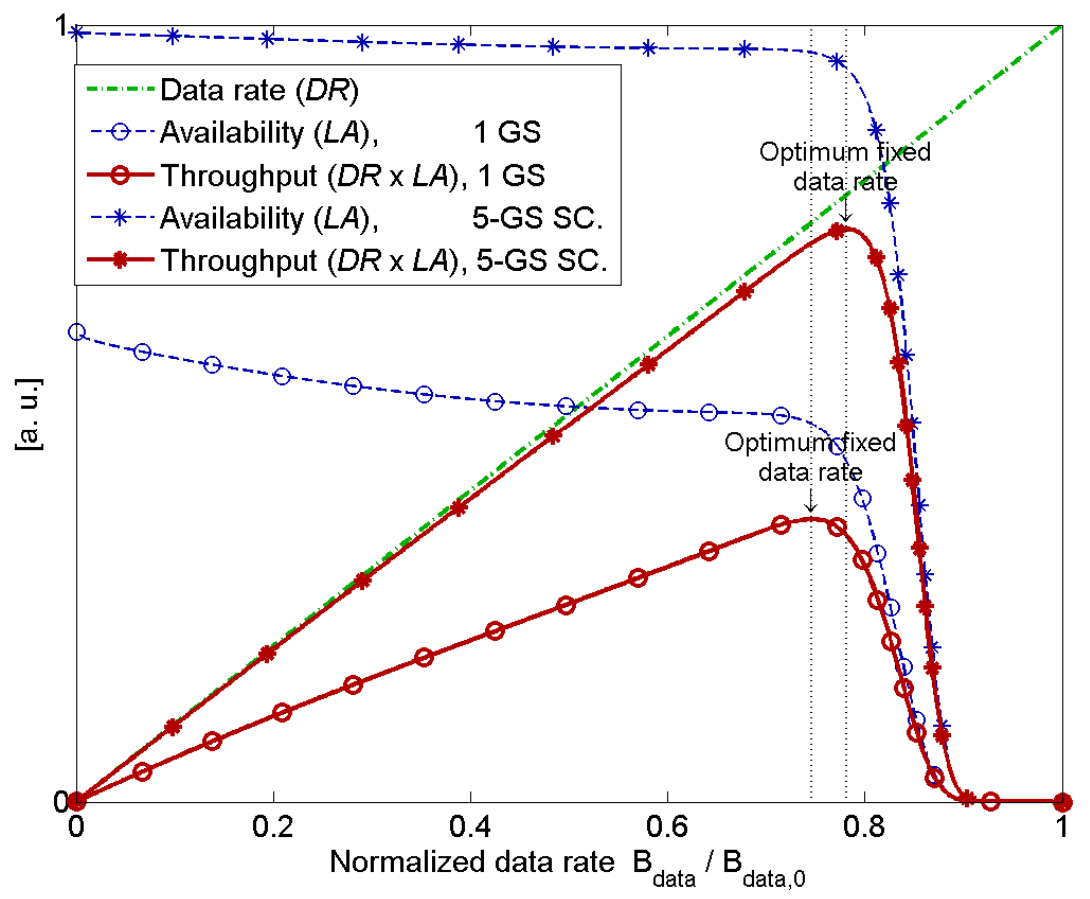

Figure 12: Illustration of the calculation of the fixed data rate that maximizes the mean throughput. The case of a single GS is compared to the case of a 5-GS network performing GS-SC.

\section{Adaptive data rate}

In the case of an adaptive data rate with infinite continuous dynamic, the throughput is the mean of the data rate variable $B_{\text {data }}$. When GS-SC is implemented, the throughput is

$$
T_{S C, A D R}=\int B_{\text {data }} f_{S C}\left(B_{\text {data }}\right) d B_{\text {data }}
$$


where $f_{S C}\left(B_{\text {data }}\right)$ is the PDF of the data rate as derived from Eq. (14). When GS-MUX is implemented we have

$$
\begin{aligned}
T_{S D M A, A D R} & =\left\langle B_{\text {data }, 1}+B_{\text {data }, 2}+\cdots+B_{\text {data }, n}\right\rangle \\
& =n\left\langle B_{\text {data }}\right\rangle \\
& =n \int B_{\text {data }} f_{B_{\text {data }}}\left(B_{\text {data }}\right) d B_{\text {data }}
\end{aligned}
$$

where $\langle\cdot\rangle$ denotes ensemble averaging and where in the second line we have assumed equal attenuation statistics for all $n$ GSs and where $f_{B_{\text {data }}}\left(B_{\text {data }}\right)$ is the data-rate PDF at an GS. The throughputs as given by Eqs. (17)-(20) are shown in Figure 13 for different GS network sizes where again we assume :

- the proportionality of the data rate with the received power (and equal data-rate capabilities for each GS).

- the same attenuation model $f_{a}(a)$ for each GS

One notes that, in the case of GS-SC, an ADR with infinite continuous dynamic increases the mean throughput by approx. $+10 \%$.

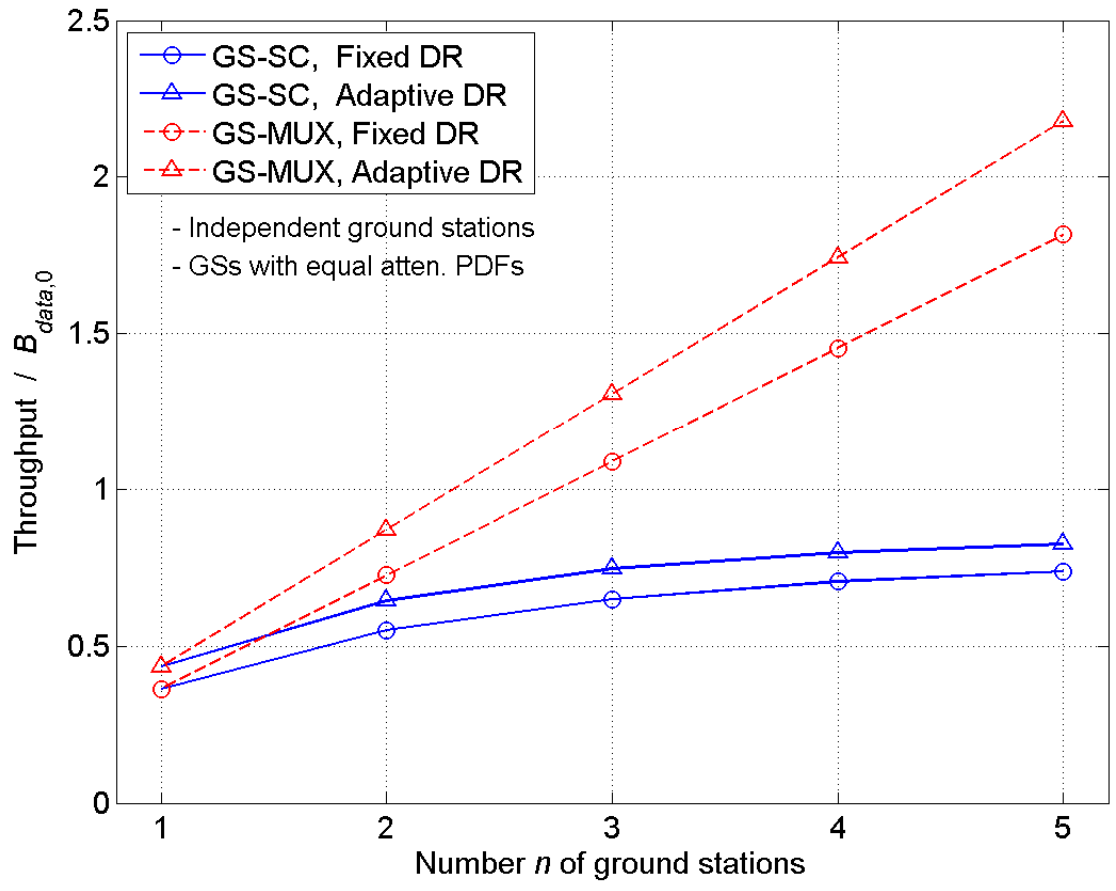

Figure 13: Mean throughput as a function of the size $n$ of the GS network. DR $=$ data rate. The continuous attenuation model at $90^{\circ}$ elevation was used.

\subsection{Discussion on Adaptive data rate}

Whereas the choice of deploying a GS network is obvious, the choice of implementing an adaptive data rate must result from a trade-off between the link availability and throughput on one hand, and the system complexity and cost on the other hand. The clear-sky attenuation dynamic is generally too small $(<6 \mathrm{~dB})$ to make ADR worthwhile: it is technically much simpler to keep the link available at a given data rate by providing a corresponding link margin. Additionally thick-cloud attenuation is too strong to allow an acceptable data rate. Consequently, ADR enhances the link quality essentially by mitigating thin clouds. As a simplification, we now limit the availability enhancement of ADR by the presence of only a thin cloud in the line of sight. Noting $p_{\text {ThickClouds }}$ the probability of having at least one thick cloud, i.e. 
$p_{\text {ThickClouds }} \equiv \operatorname{Prob}\left(E_{3}\right)$, we found that ADR reduces the LOP by the factor $\left(p_{\text {ThickClouds }} / p_{\text {cloud }}\right)^{n}$. This factor amounts, for example, to 0.4 for $p_{\text {ThickClouds }}=0.4, p_{\text {cloud }}=0.5$ and $n=4$. In the context of GS-SC, it is of interest to know how many GSs can be saved by implementing ADR if a given availability is to be reached. To this aim, we compare the availability provided by two different types of GS network: a network that mitigates thin clouds and a network that does not mitigate thin clouds. Let these two networks be composed of $n_{F D R}$ and $n_{A D R}$ GSs respectively. Their LOPs are respectively

$$
\begin{gathered}
L O P_{A D R}=p_{\text {ThickClouds }}^{n_{A \text { ARR }}} . \\
L O P_{F D R}=p_{\text {cloud }}^{n_{F D R}} .
\end{gathered}
$$

For both networks to reach the same LOPs, i.e. $L O P_{A D R}=L O P_{F D R}$, we must have

$$
p_{\text {ThickClouds }}^{n_{A D R}}=p_{\text {cloud }}^{n_{F D R}} \text {. }
$$

Noticing that we typically have $p_{\text {ThickClouds }} \approx p_{\text {cloud }}^{1.4}$, we deduce $n_{F D R} / n_{A D R} \approx 1.4$. This means that a GS network renouncing to thin-cloud mitigation would have to increase the number of GSs by a factor close to 1.4 in order to obtain the same availability.

\section{CONCLUSION}

The impact of cloud coverage on optical Earth-Space links was assessed under two attenuation models: binary and continuous. The deployment of a GS network was assessed in terms of link availability and throughput It was shown how increasing the number of GSs allows a higher link availability, and a higher throughput in case of GS-MUX. Compared to GS-SC, the throughput improvement of GS-MUX increases along with the number $n$ of stations, the clearsky probability $1-p_{\text {cloud }}$ of each GS and the level of correlation between the GSs. The continuous attenuation model allowed to assess an adaptive data rate. However ADR has limited benefits. Whether ADR should be implemented depends on the probability of thin clouds and also on the user requirements of the scenario.

\section{ACKNOWLEDGEMENT}

This work was partially funded by the European Space Agency under the ESA contract 21991/08/NL/US.

\section{REFERENCES}

[1] B. Wu, Z. Hajjarian, and M. Kavehrad, "Free space optical communications through clouds: analysis of signal characteristics," Appl. Opt. 47, 3168-3176 (2008)

[2] S. Arnon and N. S. Kopeika, "Adaptive optical transmitter and receiver for space communication through thin clouds," Appl. Opt. 36, 1987-1993 (1997)

[3] Karp, S. , R. M. Gagliardi, S. E. Moran, and L. B. Stotts. Optical Channels: Fibers, Clouds, Water, and the Atmosphere. New York: Plenum Press. 1988.

[4] A. Biswas, K.E. Wilson, S. Piazzolla, J.P. Wu, and W.H. Farr, Deep-space optical communications link availability and data volume, Proc. SPIE 5338, 175-183, 2004

[5] Garcia, P., Benarroch, A. and Riera, J. M., "Spatial distribution of cloud cover", Int. J. Satell. Commun. Network. 2008; 26:141-155

[6] A. Papoulis, Probability, Random Variables, and Stochastic Processes, $3^{\text {rd }}$ ed. (McGraw-Hill, Inc), 1991.

[7] S.S. Badesha, "SPARCL: A High Altitude Tethered Balloon-Based Optical Space-to-Ground Communication System", Proceedings of SPIE, vol. 4821, pp. 181-193, 2002

[8] University of Washington, Department of Atmospheric Sciences. http://www.atmos.washington.edu/ ignatius/CloudMap/, 2006. 